\title{
Comparative effectiveness of upfront esophagectomy versus induction chemoradiation in clinical stage T2N0 esophageal cancer: A decision analysis
}

\author{
Tara R. Semenkovich, MD, MPHS, ${ }^{a}$ Roheena Z. Panni, MD, Jessica L. Hudson, MD, MPHS, ${ }^{\text {a }}$ \\ Theodore Thomas, MD, MPHS, ${ }^{\mathrm{c}}$ Leisha C. Elmore, MD, MPHS, ${ }^{\mathrm{b}}$ Su-Hsin Chang, PhD, ${ }^{\mathrm{d}}$ \\ Bryan F. Meyers, MD, MPH, ${ }^{a}$ Benjamin D. Kozower, MD, MPH, ${ }^{a}$ and Varun Puri, MD, MSCI ${ }^{\mathrm{a}}$
}

\section{ABSTRACT}

Objectives: We compared the effectiveness of upfront esophagectomy versus induction chemoradiation followed by esophagectomy for overall survival in patients with clinical T2N0 (cT2N0) esophageal cancer. We also assessed the influence of the diagnostic uncertainty of endoscopic ultrasound on the expected benefit of chemoradiation.

Methods: We created a decision analysis model representing 2 treatment strategies for cT2N0 esophageal cancer: upfront esophagectomy that may be followed by adjuvant therapy for upstaged patients and induction chemoradiation for all patients with cT2N0 esophageal cancer followed by esophagectomy. Parameter values within the model were obtained from published data, and median survival for pathologic subgroups was derived from the National Cancer Database. In sensitivity analyses, staging uncertainty of endoscopic ultrasound was introduced by varying the probability of pathologic upstaging.

Results: The baseline model showed comparable median survival for both strategies: 48.3 months for upfront esophagectomy versus 45.9 months for induction chemoradiation and surgery. The sensitivity analysis demonstrated induction chemoradiation was beneficial, with probability of upstaging $>48.1 \%$, which is within the published range of $32 \%$ to $65 \%$ probability of pathologic upstaging after cT2N0 diagnosis. The presence of any of 3 key variables (size larger than $3 \mathrm{~cm}$, high grade, or lymphovascular invasion) was associated with $>48.1 \%$ risk of upstaging, thus conferring a survival advantage to induction chemoradiation.

Conclusions: The optimal treatment strategy for cT2N0 esophageal cancer depends on the accuracy of endoscopic ultrasound staging. High-risk features that confer increased probability of upstaging can inform clinical decision making to recommend induction chemoradiation for select cT2N0 patients. (J Thorac Cardiovasc Surg 2018;155:2221-30)

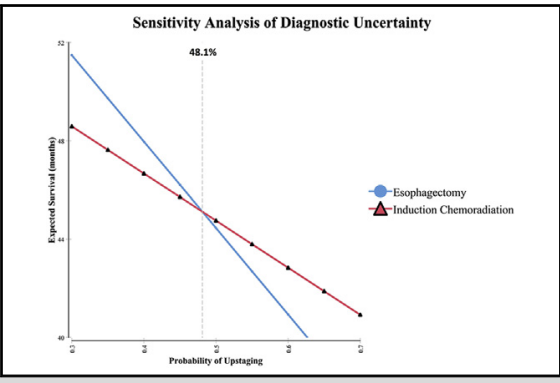

Induction chemoradiation is beneficial with a $48.1 \%$ or higher probability of upstaging.

\section{Central Message}

Induction chemoradiation may offer a survival benefit over upfront surgery for patients with clinical T2NO esophageal cancer who have $>48 \%$ probability of pathologic upstaging.

\section{Perspective}

This research aims to address the controversy in treating cT2N0 esophageal cancer that exists because of the inaccuracies in endoscopic ultrasound staging. We created a decision analysis model to compare 2 treatment paradigms for patients with cT2NO esophageal cancer: upfront esophagectomy and induction chemoradiation followed by esophagectomy, focusing on the influence of diagnostic inaccuracy on patient outcomes.

See Editorial Commentary page 2231
Esophageal cancer is a common malignancy in the United States, with approximately 17,000 new cases annually. ${ }^{1}$ Disease stage is based on the American Joint Committee

\footnotetext{
From the Divisions of ${ }^{\mathrm{a}}$ Cardiothoracic Surgery and ${ }^{\mathrm{b}}$ Surgical Oncology, Department of Surgery, ${ }^{\mathrm{c}}$ Division of Oncology, Department of Medicine, and ${ }^{\mathrm{d}}$ Division of Public Health Sciences, Department of Surgery, Washington University, St Louis, Mo. Dr Semenkovich was supported by National Institutes of Health (NIH) grant No. 2T32HL7776-21. Drs Panni and Elmore were supported by NIH grant No. 5T32CA00962128. Dr Hudson was supported by NIH grant No. 5T32CA009621-27. Dr Chang was supported by Agency for Healthcare Research and Quality grant No. K01 HS022330 and NIH grant No. R21 DK110530. Dr Puri was supported by NIH grant No. K07 CA178120.
}

on Cancer criteria ${ }^{2}$ and determines the treatment paradigm. Treatment for early stage, localized disease (T1N0) is primary surgery with no proven benefit from induction 


\section{Abbreviations and Acronyms \\ CROSS $=$ Chemoradiotherapy for Oesophageal Cancer Followed by Surgery Study \\ EUS = endoscopic ultrasound \\ $\mathrm{NCDB}=$ National Cancer Database}

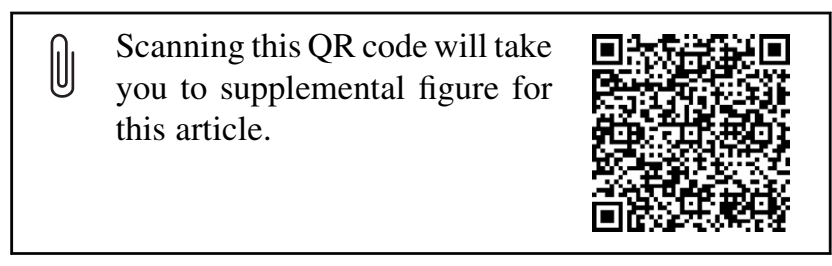

therapy, and possibly a detriment in outcomes. ${ }^{3,4}$ Patients with locally advanced disease (T2-T4, N1-N3) have improved outcomes with induction chemoradiotherapy compared with surgery alone. ${ }^{5}$ There are conflicting data from retrospective studies on the benefit of neoadjuvant chemoradiation for clinical T2N0 (cT2N0) disease., ${ }^{4-9}$ This observed variability in results may be due to inaccuracies in clinical staging.

Endoscopic ultrasound (EUS) is the key diagnostic tool used for determining clinical stage in locoregional disease by assessing the depth of tumor invasion and presence of regional lymphatic spread. Clinical EUS findings have been compared with pathologic staging in surgical resection specimens and accuracy varies drastically by stage. ${ }^{10}$ In patients with early and locally advanced disease, EUS has a sensitivity of $75 \%$ and $73.7 \%$, respectively, for properly identifying the stage of disease. ${ }^{10}$ In patients with cT2N0 disease, EUS performs poorly with a sensitivity of only $9.1 \% .{ }^{10}$ Several series have found that cT2N0 patients truly had pathologic T2N0 (pT2N0) disease only $6 \%$ to $29 \%$ of the time, with probabilities of a true higher stage ranging from $32 \%$ to $65 \%$. ${ }^{4,8-16}$ These pathologically upstaged patients were actually understaged clinically with EUS. Because clinical staging is crucial in selecting appropriate treatments to maximize survival, diagnostic uncertainty in cT2N0 esophageal cancer has led to controversy about the optimal treatment strategy. Consequently, clinical practice with regard to providing induction therapy versus upfront surgery is equivocal. ${ }^{8,16}$

The gold standard for determining the optimal treatment for cT2NO patients might be to conduct a randomized controlled trial. Conducting such a large-scale trial in this limited subset of patients with esophageal cancer would be costly, accrue patients slowly, and require long-term follow-up, making a clinical trial a very difficult way to answer this question. Therefore, to address this problem, we created a decision analysis model to evaluate the role of neoadjuvant therapy versus upfront resection in cT2N0 patients with esophageal cancer and to identify the threshold for benefit of induction chemoradiation. We explored the effect of the diagnostic uncertainty of EUS and operative risk on the model, and evaluated tumor characteristics that affect a patient's probability of upstaging.

\section{METHODS}

\section{Decision Analysis Model}

To determine the optimal strategy for treatment of patients with cT2N0 esophageal cancer, we developed a decision analysis model using TreeAge Pro software (version R2.1; TreeAge Inc, Williamstown, Mass). The model compares survival based on initial treatment with surgery or chemoradiation. Decision analysis uses mathematical modeling to compare different treatment modalities by modeling plausible outcomes over a range of clinical scenarios, estimating the effectiveness of each treatment strategy. Sensitivity analyses can be performed to incorporate uncertainty and assess the driving factors in the model. The probabilities and median survival used to populate the decision tree were obtained from published literature and a large validated database, the National Cancer Database (NCDB).

\section{Treatment Strategies and Structure of the Model}

We modeled 2 groups of cT2N0 patients eligible for surgery: upfront esophagectomy that may be followed by adjuvant therapy for pathologically upstaged patients and induction therapy followed by esophagectomy. The model evaluated which treatment strategy would yield a better overall survival. These 2 general treatment paradigms are reflected in our decision tree (Figure 1). Patients in the esophagectomy arm were dichotomized to perioperative death (defined as death within 30 days of surgery) or survival. Patients surviving esophagectomy may receive adjuvant systemic therapy if they have locally advanced disease (eg, $\mathrm{T} 3$ or $\mathrm{N}+$ ). In contrast, patients in the induction chemoradiation arm may experience death related to chemoradiation, become a nonoperative candidate due to treatment-related morbidity or progression of disease, or they may remain an operative candidate and proceed to esophagectomy.

\section{Model Parameters and Data Sources}

The baseline values used in the model are described in the following paragraphs and are summarized in Table 1.

\section{Perioperative Mortality}

Perioperative outcomes were assessed using 30-day mortality estimated at $3 \%{ }^{13}$ in both the upfront esophagectomy and induction chemoradiation arms (Figure 1). In patients who died within the perioperative period after upfront surgery, survival was counted as 0 months. However, patients receiving induction chemoradiation have additional survival time during neoadjuvant chemoradiation before perioperative death at time of esophagectomy. Patients dying during the perioperative period after induction therapy were assigned a median survival of 2.4 months to account for chemoradiation therapy and the standard delay following induction therapy before surgery based on the findings in Preoperative Chemoradiotherapy for Esophageal or Junctional Cancer (Chemoradiotherapy for Oesophageal Cancer Followed by Surgery Study [CROSS] trial). ${ }^{5}$

\section{Morbidity and Mortality of Induction Therapy}

Mortality during induction chemoradiation in our baseline model was $0.6 \%$ based on the CROSS trial. ${ }^{5}$ Patients who had a death related to induction therapy were assigned a survival of 0 months. Patients receiving induction therapy may not be operative candidates after completion of therapy. Based on CROSS trial data, we used an estimate of $6 \%$ in our baseline model to represent the proportion of patients who underwent 


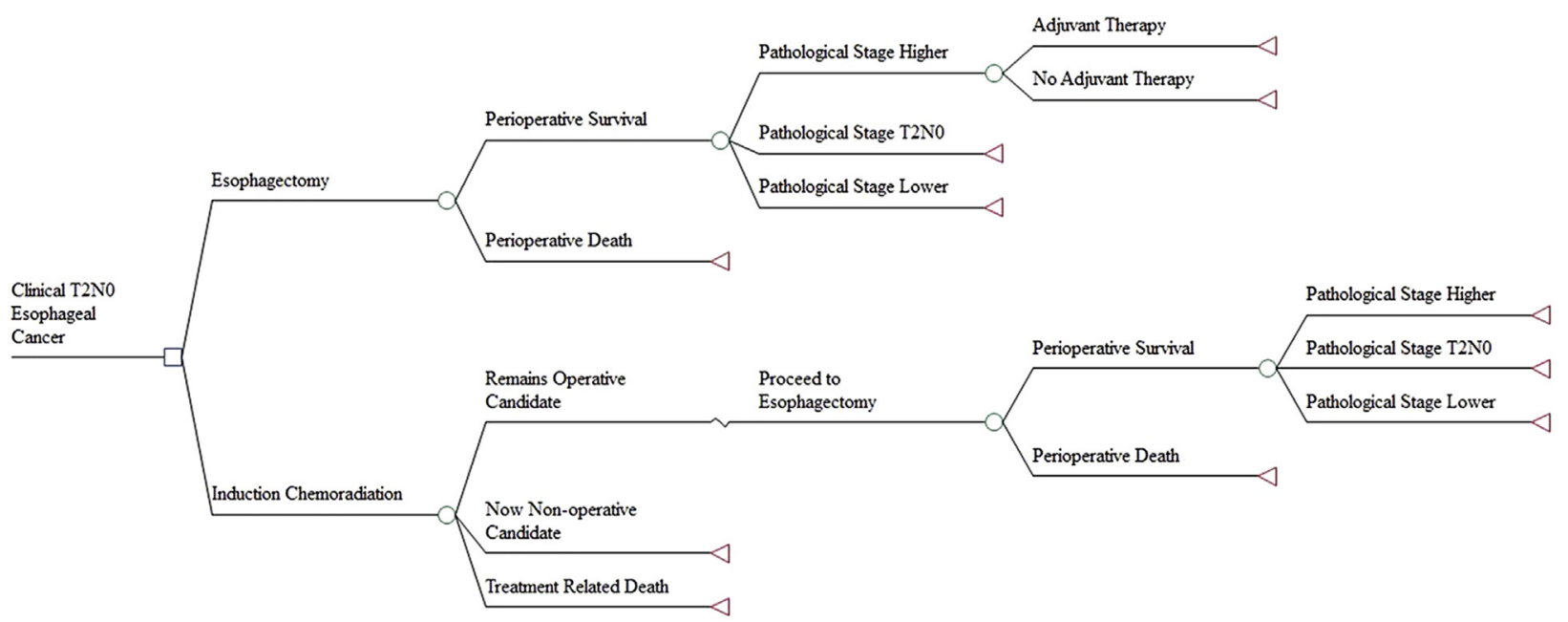

FIGURE 1. Decision tree comparing upfront esophagectomy to induction chemoradiation followed by esophagectomy.

chemoradiation and were no longer operative candidates because of progression of disease, patient preference, toxicity of chemotherapy, or decline in health making them medically unfit for surgery. ${ }^{5}$ Median survival for patients who were deemed inoperable after induction therapy was 16 months. ${ }^{17}$

\section{Accuracy of Clinical Staging}

To reflect the uncertainty in EUS and the influence on survival in the esophagectomy arm, we included the probability of upstaging, downstaging, and accurate staging using pathology data in patients who had undergone upfront esophagectomy. A literature search revealed 10 studies that examined the diagnostic accuracy of endoscopic ultrasound in cT2N0 cancers. A summary of these studies, the number of patients included, and the proportions of patients with same, down-, or upstaged disease is included in Table 2. For our baseline analysis, the largest study by Speicher and colleagues $^{8}$ was used, and the probabilities of same, up- and downstaging were set at $0.416,0.267$, and 0.317 , respectively. As shown in Table 2, the range of upstaging probability ranged from $32 \%$ to $65 \%$, and this variability was used as the basis for our 1-way sensitivity analysis of diagnostic uncertainty (Figure 2). Because we varied the probability of upstaging in our model from 0.3 to 0.7 to encompass the entire published range, we held the ratio of the remaining proportion of same- and downstaged

TABLE 1. Probabilities used in the baseline decision-analysis model

\begin{tabular}{|c|c|c|}
\hline Variable description & Data source & Value \\
\hline \multicolumn{3}{|l|}{ Upfront esophagectomy } \\
\hline Probability of preoperative death & Hofstetter and colleagues $(2014)^{13}$ & 0.03 \\
\hline Probability of patient upstaging & Speicher and colleagues $(2014)^{8}$ & 0.416 \\
\hline Probability of pathologically staged $\mathrm{T} 2 \mathrm{~N} 0$ & Speicher and colleagues $(2014)^{8}$ & 0.267 \\
\hline Probability of patient downstaging & Speicher and colleagues $(2014)^{8}$ & 0.317 \\
\hline Probability of receiving adjuvant therapy if upstaged & Speicher and colleagues $(2014)^{8}$ & 0.50 \\
\hline Survival with adjuvant therapy in upstaged patients & Samson and colleagues $(2016)^{16}$ & $34.6 \mathrm{mo}$ \\
\hline Survival with surgery only in upstaged patients & Samson and colleagues $(2016)^{16}$ & $20.8 \mathrm{mo}$ \\
\hline Survival with surgery only in pathologically staged $\mathrm{T} 2 \mathrm{~N} 0$ patients & Derived from NCDB & $44.4 \mathrm{mo}$ \\
\hline Survival with surgery only in downstaged patients & Derived from NCDB & $83.6 \mathrm{mo}$ \\
\hline \multicolumn{3}{|l|}{ Induction chemoradiation followed by esophagectomy } \\
\hline Probability that patient becomes a nonoperative candidate after induction therapy & van Hagen and colleagues $(2012)^{5}$ & 0.06 \\
\hline Probability of chemoradiation-related death & van Hagen and colleagues $(2012)^{5}$ & 0.006 \\
\hline Probability of postinduction higher pathologic stage & Speicher and colleagues $(2014)^{8}$ & 0.341 \\
\hline Probability of postinduction pathologically staged $\mathrm{T} 2 \mathrm{NO}$ & Speicher and colleagues $(2014)^{8}$ & 0.243 \\
\hline Probability of postinduction lower pathologic stage & Speicher and colleagues $(2014)^{8}$ & 0.416 \\
\hline Probability of postinduction perioperative death & Hofstetter and colleagues $(2014)^{13}$ & 0.03 \\
\hline Survival if patient has a perioperative death after induction & van Hagen and colleagues $(2012)^{5}$ & $2.3 \mathrm{mo}$ \\
\hline Survival if patient becomes a nonoperative candidate & Abrams and colleagues $(2009)^{17}$ & $16.0 \mathrm{mo}$ \\
\hline Survival with trimodality therapy in upstaged patients & Derived from NCDB & $34.6 \mathrm{mo}$ \\
\hline Survival with trimodality therapy in postinduction pathologically staged $\mathrm{T} 2 \mathrm{~N} 0$ patients & Derived from NCDB & $43.6 \mathrm{mo}$ \\
\hline Survival with trimodality therapy in downstaged patients & Derived from NCDB & $66.5 \mathrm{mo}$ \\
\hline
\end{tabular}


TABLE 2. Published diagnostic accuracy of endoscopic ultrasound in patients staged as clinical T2N0 cancer undergoing upfront surgery. In patients undergoing upfront surgery, pathologic upstaging is equivalent to esophageal ultrasound understaging and means that the patient was found to have T3 or T4 or N+ disease following surgical resection. Pathologic downstaging is equivalent to esophageal ultrasound overstaging and means the patient had T1N0 disease

\begin{tabular}{|c|c|c|c|c|}
\hline Study & $\mathbf{n}$ & Probability of pT2NO & $\begin{array}{c}\text { Probability of } \\
\text { pathologic downstaging }\end{array}$ & $\begin{array}{c}\text { Probability of } \\
\text { pathologic upstaging }\end{array}$ \\
\hline Rice and colleagues $(2007)^{4}$ & 53 & $13.0 \%$ & $55.0 \%$ & $32.0 \%$ \\
\hline Crabtree and colleagues $(2011)^{10}$ & 18 & $6.0 \%$ & $50.0 \%$ & $44.0 \%$ \\
\hline Stiles and colleagues $(2011)^{11}$ & 40 & $12.5 \%$ & $22.5 \%$ & $65.0 \%$ \\
\hline Zhang and colleagues (2012) ${ }^{9}$ & 14 & $29.0 \%$ & $21.0 \%$ & $50.0 \%$ \\
\hline Crabtree and colleagues $(2013)^{12}$ & 752 & $27.4 \%$ & $25.9 \%$ & $46.7 \%$ \\
\hline Hofstetter and colleagues $(2014)^{13}$ & 499 & $14.0 \%$ & $44.0 \%$ & $42.0 \%$ \\
\hline Hardacker and colleagues $(2014)^{14}$ & 68 & $8.5 \%$ & $42.8 \%$ & $48.5 \%$ \\
\hline Speicher and colleagues $(2014)^{8}$ & 1599 & $26.7 \%$ & $31.7 \%$ & $41.6 \%$ \\
\hline Tekola and colleagues $(2014)^{15}$ & 38 & $21.0 \%$ & $47.0 \%$ & $32.0 \%$ \\
\hline Samson and colleagues $(2016)^{16}$ & 932 & \multicolumn{2}{|c|}{$54.3 \%$} & $45.7 \%$ \\
\hline Range & & $6 \%-29 \%$ & $21 \%-55 \%$ & $32 \%-65 \%$ \\
\hline
\end{tabular}

patients at $1: 1$. This reflected the ratios seen in the largest clinical studies $^{8,12}$ and prevented an unrealistic elimination of a pathologic subgroup at the high end of the sensitivity analysis.

\section{Treatment Effect of Induction Therapy}

To evaluate the treatment effect of induction therapy, pathologic stage after trimodality treatment with induction chemotherapy and radiation followed by esophagectomy was included in our model. For our baseline analysis, the largest study by Speicher and colleageus ${ }^{8}$ was used, and the probabilities of upstaging, same stage, or downstaging for the induction therapy arm were set at $0.341,0.243$, and 0.416 , respectively. These probabilities reflect the both the diagnostic uncertainty of EUS as well as the treatment benefit of induction therapy because fewer patients are likely to be upstaged compared with upfront surgery. When conducting our sensitivity analysis of diagnostic uncertainty, we held the ratio of patients upstaged after upfront surgery to patients upstaged after induction therapy and surgery constant at 0.82 , based on the ratio observed in the study by Speicher and colleagues. ${ }^{8}$ Additionally, we held the ratio of the remaining patients with the same stage versus downstaged stable at 1:2. This incorporated the expected treatment effect of chemoradiation in a realistic way, preventing distortion of the probabilities of each outcome as the chance of upstaging was varied.

\section{Adjuvant Therapy Use}

Patients with cT2N0 disease who undergo upfront esophagectomy and are upstaged are candidates for adjuvant chemoradiation. Practice patterns for the use of adjuvant therapy are inconsistent. We used data from multiple NCDB studies that demonstrated, in current practice, approximately $50 \%$ of upstaged cT2N0 patients receive adjuvant therapy. ${ }^{8,16}$

\section{Survival Outcomes for Patients in Each Arm}

For upstaged patients undergoing upfront esophagectomy, median survival data were included from a study by Samson and colleagues ${ }^{16}$ that explicitly examined upstaged cT2N0 patients stratified based on receipt of adjuvant therapy. Those receiving adjuvant therapy had a median survival of 34.6 months versus 20.8 months for surgery alone, and these values were incorporated into our model. For the remaining arms, no published data were available that identified median survival for individual groups of patients with cT2N0 disease treated with upfront surgery or induction chemoradiation separated by their ultimate pathologic stage. Therefore, we used data from the NCDB to generate Kaplan-Meier survival curves using SAS software version 9.4 (SAS System for Windows, SAS Institute Inc, Cary, NC). The survival curves can be seen in Figure E1. Median survival estimates resulting from these curves were 44.4 months for pT2N0 disease after upfront surgery, 83.6 months for downstaged patients after upfront surgery, 34.6 months for patients pathologically upstaged despite receiving induction therapy, 43.6 months for postinduction pathologically staged T2N0 disease, and 66.5 months for downstaged patients after induction chemoradiation.

\section{Decision Analysis and Sensitivity Analyses}

The decision analysis model was run using TreeAge Pro to compute expected survival time for each treatment paradigm to determine which yields the longest expected length of survival, incorporating the evidence-based input probabilities for entering each branch point with median survival as the outcome of interest for the terminal node. All of the baseline characteristics described above are listed in Table 1.

Within decision analysis, sensitivity analyses assess the stability of the model over a range of probabilities, exploring how the assumptions made with the various baseline inputs influence the overall outcome of the model. One-way sensitivity analysis was conducted on the probability of upstaging in the esophagectomy arm as described above to incorporate the diagnostic uncertainty of EUS. Additionally, we performed a 1-way sensitivity analysis examining operative risk by varying the probability of perioperative mortality between $3 \%$ and $10 \%$, a clinically plausible range. ${ }^{13,18,19} \mathrm{We}$ also evaluated the effect of differential perioperative mortality between the 2 arms. Despite experienced centers reporting comparable mortality rates between the 2 strategies, ${ }^{13}$ a meta-analysis estimated patients treated with chemoradiation may have twice the perioperative mortality as patients receiving surgery alone, ${ }^{20}$ so we varied the mortality rate up to $6 \%$ in the induction therapy arm.

\section{Identifying Features That Predict Upstaging}

After identifying the threshold of EUS understaging at which a benefit would be expected from induction chemoradiation, we sought to investigate clinical or pathologic features of patients or their tumors that would place them at risk of pathologic upstaging higher than the identified threshold, suggesting these individuals might benefit from induction 
chemoradiation. To do this, we analyzed patients with cT2N0 disease from the NCDB who underwent upfront surgery from 1998 to 2012. Descriptive statistics were performed comparing tumor characteristics (eg, grade, histology, lymphovascular invasion, and tumor size) and patient characteristics (eg, age, sex, race, urban/rural, cancer center type, insurance status, education status by ZIP code, income quartile by ZIP code, and Charlson Deyo score) by pathologic staging group with $\chi^{2}$, Kruskal-Wallis, and analysis of variance tests. Univariable and multivariable analyses were performed using binary logistic regression to assess the possible predictive value for each of these variables for upstaging versus same stage or downstaging.

\section{RESULTS}

\section{Baseline Analysis}

Our model resulted in an expected survival of 48.3 months in the patients receiving upfront esophagectomy compared with 45.9 months in patients treated with induction chemoradiation, yielding an estimated survival benefit of approximately 2.5 months with the upfront surgery strategy. To restate in another way, routine application of induction therapy was likely to be harmful rather than beneficial with regard to survival.

\section{Threshold Analysis for EUS Diagnostic Uncertainty}

The proportion of patients likely to be upstaged, and therefore benefit most from induction chemoradiation, across many published studies ranges from $32 \%$ to $65 \%$ as shown in Table 2 . We varied this parameter in the 1-way sensitivity analysis while holding the ratio of same and downstaged patients stable at 1:1. The results of this analysis (Figure 2) revealed that with a probability of upstaging of $48.1 \%$ or greater, induction chemoradiation would be expected to confer a survival benefit over upfront esophagectomy.

\section{Sensitivity Analysis for Perioperative Mortality}

In another 1-way sensitivity analysis, we varied the probability of perioperative mortality up to $10 \%$, keeping all other inputs stable. The results showed that upfront esophagectomy is the favored strategy compared with neoadjuvant treatment with a survival benefit of 2 to 3 months throughout this range of surgical risk. When we evaluated differential risk in perioperative mortality between the 2 strategies by increasing the rate in the induction arm, we found that the threshold of pathologic upstaging for benefit of induction chemoradiation increased to $50.8 \%, 53.4 \%$, and $55.9 \%$ at $4 \%, 5 \%$, and $6 \%$ mortality, respectively.

\section{Identifying Features That Predict Upstaging}

Tumor and patient characteristics were examined in 1520 patients in the NCDB with cT2N0 disease who underwent upfront surgery and had available pathologic staging data. These characteristics were compared across patients who were pathologically downstaged, pT2N0, and upstaged

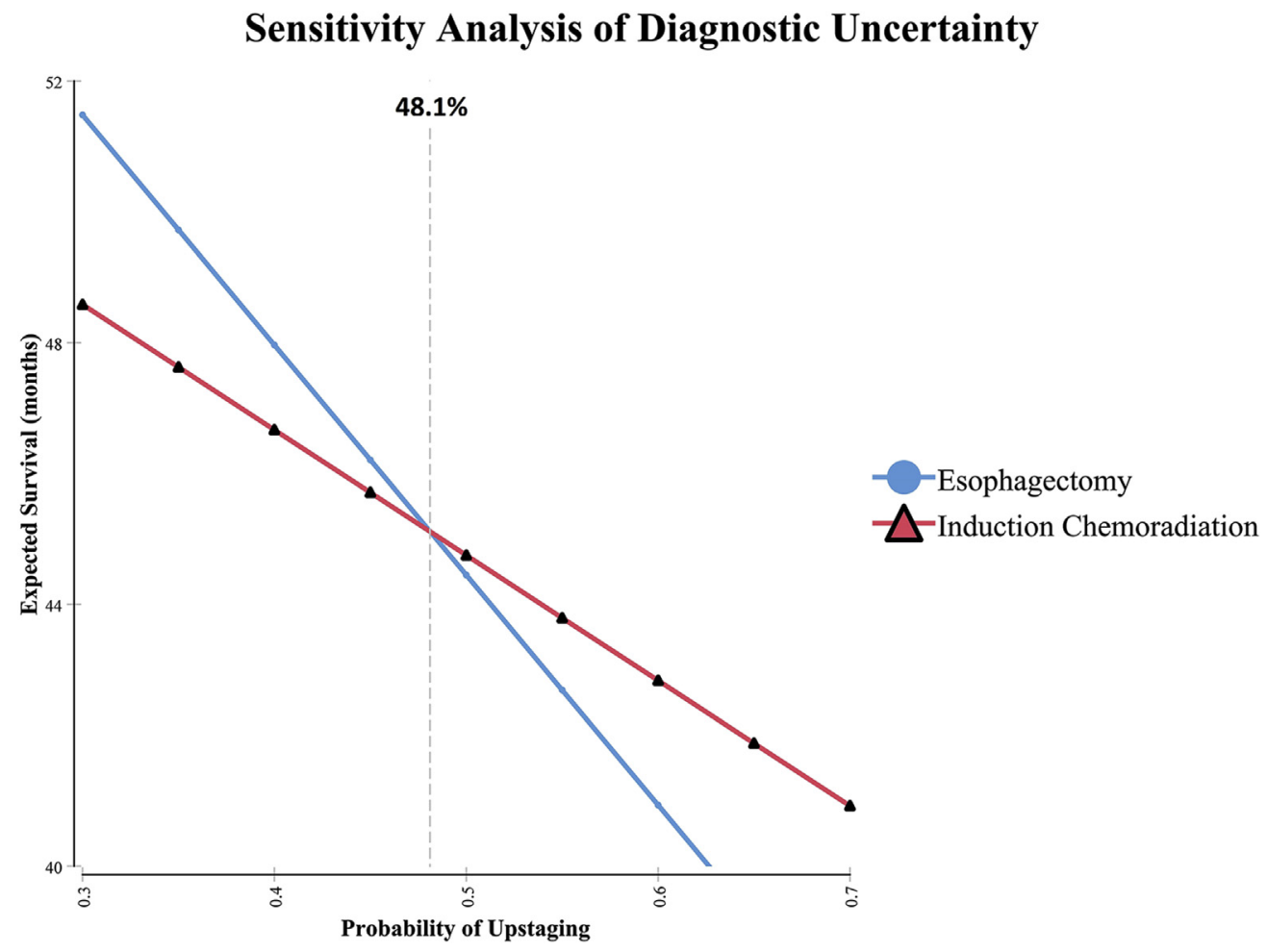

FIGURE 2. One-way sensitivity analysis varying the probability of upstaging between $30 \%$ and $70 \%$, with a threshold for benefit of induction chemoradiation seen at $48.1 \%$. 
and the results of selected descriptive comparisons can be seen in Table 3. Tumor grade $(P<.001)$, histology $(P<.001)$, lymphovascular invasion $(P<.001)$, tumor size $(P<.001)$, sex $(P=.008)$, cancer center type $(P<.001)$, and education status $(P=.013)$ were significantly different between the pathologic staging groups. Age, race, urban/rural, insurance status, income quartile by ZIP code, and Charlson Deyo score were not significantly different between groups.
Of the features that were statistically significant, tumor grade, presence of lymphovascular invasion, and tumor size all had clinically meaningful categories that placed a patient above the threshold of $48.1 \%$ probability of upstaging, where patients may be expected to benefit from routine induction chemoradiation. These categories are shown in Table 3. For patients with tumor grade 3 and $4,54.2 \%$ and $55.6 \%$, respectively, were ultimately pathologically upstaged. Patients with evidence of lymphovascular invasion

TABLE 3. Characteristics of patients in the National Cancer Database with cT2N0 esophageal cancer undergoing upfront surgery by true pathologic stage

\begin{tabular}{|c|c|c|c|c|c|}
\hline Variable & n ( $\%$ of total) & Downstaged & True T2No & Upstaged & $P$ value \\
\hline \multicolumn{6}{|l|}{ Tumor characteristic } \\
\hline Grade & & & & & $<.001$ \\
\hline 1 & $94(6.2)$ & $32(34.0)$ & $47(50.0)$ & $15(16.0)$ & \\
\hline 2 & $644(42.4)$ & $153(23.8)$ & $243(37.8)$ & $248(38.5)$ & \\
\hline 3 & $638(42.0)$ & $83(13.1)$ & $209(32.8)$ & $346(54.2)^{*}$ & \\
\hline 4 & $27(1.8)$ & $5(18.5)$ & $7(25.9)$ & $15(55.6)^{*}$ & \\
\hline Missing & $117(7.7)$ & $37(31.6)$ & $43(36.7)$ & $37(31.6)$ & \\
\hline Histology & & & & & $<.001$ \\
\hline Squamous & $324(21.3)$ & $55(17.0)$ & $137(42.3)$ & $132(40.7)$ & \\
\hline Adenocarcinoma & 1088 (71.6) & $245(22.5)$ & $376(34.6)$ & $467(42.9)$ & \\
\hline Other & $108(7.1)$ & $10(9.2)$ & $36(33.3)$ & $62(57.4)$ & \\
\hline Lymphovascular invasion & & & & & $<.001$ \\
\hline Present & $92(6.1)$ & $2(2.2)$ & $17(18.5)$ & $73(79.4)^{*}$ & \\
\hline Absent & $137(9.0)$ & $12(8.8)$ & $61(44.5)$ & $64(46.7)$ & \\
\hline Missing & $1291(85.0)$ & $296(22.9)$ & $471(36.5)$ & $524(40.6)$ & \\
\hline Tumor size $(\mathrm{cm})$ & & & & & $<.001$ \\
\hline Missing & $259(17.0)$ & $68(26.2)$ & $104(40.2)$ & $87(33.6)$ & \\
\hline$<1$ & $74(4.9)$ & $29(39.2)$ & $33(44.6)$ & $12(16.2)$ & \\
\hline 1 to $<2$ & $241(15.9)$ & $74(30.7)$ & $88(36.5)$ & $79(32.8)$ & \\
\hline 2 to $<3$ & $329(21.6)$ & $59(17.9)$ & $118(35.6)$ & $152(46.2)$ & \\
\hline 3 to $<4$ & $250(16.4)$ & $42(16.8)$ & $85(34.0)$ & $123(49.2)^{*}$ & \\
\hline 4 to $<5$ & 267 (17.6) & $20(12.0)$ & $50(29.9)$ & $97(58.1)^{*}$ & \\
\hline $5+$ & $200(13.2)$ & $18(9.0)$ & $71(35.5)$ & $111(55.5)^{*}$ & \\
\hline \multicolumn{6}{|l|}{ Patient characteristic } \\
\hline Age & & & & & .585 \\
\hline Mean \pm standard deviation & & $64.4 \pm 10.0$ & $65.1 \pm 11.1$ & $64.5 \pm 11.0$ & \\
\hline Median (interquartile range) & & $65.5(58-72)$ & $66(57-74)$ & $65(58-73)$ & \\
\hline Sex & & & & & .005 \\
\hline Male & $1234(81.2)$ & $264(21.4)$ & $424(34.4)$ & $546(44.3)$ & \\
\hline Female & $286(18.8)$ & $46(16.1)$ & $125(43.7)$ & $115(40.2)$ & \\
\hline Race & & & & & .847 \\
\hline White & 1393 (91.6) & 287 (20.6) & $500(35.9)$ & $606(43.5)$ & \\
\hline Black & $77(5.1)$ & $12(15.6)$ & $31(40.3)$ & $34(44.2)$ & \\
\hline Other & $50(3.3)$ & $11(22.0)$ & $18(36.0)$ & $21(42.0)$ & \\
\hline Urban/rural county & & & & & .110 \\
\hline Metropolitan & $1169(76.9)$ & $242(20.7)$ & $424(36.7)$ & $503(43.0)$ & \\
\hline Urban & $230(15.1)$ & $34(14.8)$ & $84(36.5)$ & $112(48.7)$ & \\
\hline Rural & $30(2.0)$ & $10(33.3)$ & $9(30.0)$ & $11(36.7)$ & \\
\hline Cancer center type & & & & & $<.001$ \\
\hline Community & $674(44.3)$ & $112(16.6)$ & $272(40.4)$ & $290(43.0)$ & \\
\hline Academic & $846(55.7)$ & $198(23.4)$ & $277(32.7)$ & $371(43.9)$ & \\
\hline
\end{tabular}

*Tumor feature associated with upstaging. Presence of these features place patients above the $48.1 \%$ probability of upstaging threshold where they may benefit from routine induction chemoradiation. 
TABLE 4. Univariable and multivariable analysis of selected covariates with likelihood of upstaging as the dependent variable

\begin{tabular}{|c|c|c|c|c|}
\hline \multirow[b]{2}{*}{ Variable } & \multicolumn{2}{|l|}{ Univariable analysis } & \multicolumn{2}{|l|}{ Multivariable analysis } \\
\hline & Odds ratio $(95 \%$ confidence interval) & $P$ value & $\overline{\text { Odds ratio }(95 \% \text { confidence interval) }}$ & $\overline{P \text { value }}$ \\
\hline \multicolumn{5}{|l|}{ Tumor characteristic } \\
\hline Grade & & $<.0001$ & & $<.0001$ \\
\hline 1 & Reference & & Reference & \\
\hline 2 & $3.3(1.9-5.9)$ & & $2.6(1.3-5.1)$ & \\
\hline 3 & $6.2(3.5-11.1)$ & & $5.6(2.9-10.8)$ & \\
\hline 4 & $6.6(2.6-16.8)$ & & $3.8(1.4-10.9)$ & \\
\hline Missing & $2.4(1.2-4.8)$ & & $4.2(1.9-9.5)$ & \\
\hline Histology & & .009 & & \\
\hline Squamous & $0.9(0.7-1.2)$ & & & \\
\hline Adenocarcinoma & Reference & & & \\
\hline Other & $1.7(1.2-2.7)$ & & & \\
\hline Lymphovascular invasion & & $<.0001$ & & $<.0001$ \\
\hline Present & $5.6(3.4-9.4)$ & & $4.6(2.6-7.9)$ & \\
\hline Absent & $1.3(0.9-1.8)$ & & $1.4(1.0-2.1)$ & \\
\hline Missing & Reference & & Reference & \\
\hline Tumor size $(\mathrm{cm})$ & & $<.0001$ & & $<.0001$ \\
\hline$<1$ & Reference & & Reference & \\
\hline 1 to $<2$ & $2.5(1.3-4.9)$ & & $3.1(1.4-6.6)$ & \\
\hline 2 to $<3$ & $4.4(2.3-8.5)$ & & $5.3(2.5-11.0)$ & \\
\hline 3 to $<4$ & $5.0(2.6-9.7)$ & & $5.5(2.6-11.6)$ & \\
\hline 4 to $<5$ & $7.2(3.6-14.3)$ & & $7.9(3.6-17.0)$ & \\
\hline $5+$ & $6.4(3.3-12.7)$ & & $7.3(3.4-15.7)$ & \\
\hline \multicolumn{5}{|l|}{ Patient characteristic } \\
\hline Age & $1.0(0.99-1.01)$ & .531 & & \\
\hline \multicolumn{5}{|l|}{ Sex } \\
\hline Male vs female & $1.2(0.9-1.5)$ & .215 & & \\
\hline \multicolumn{5}{|l|}{ Race } \\
\hline White & Reference & .971 & & \\
\hline Black & $1.0(0.6-1.6)$ & & & \\
\hline Other & $0.9(0.5-1.7)$ & & & \\
\hline \multicolumn{5}{|l|}{ Urban or rural county } \\
\hline Metropolitan & Reference & .209 & & \\
\hline Urban & $1.3(0.9-1.7)$ & & & \\
\hline Rural & $0.8(0.4-1.6)$ & & & \\
\hline \multicolumn{5}{|l|}{ Cancer center } \\
\hline Academic vs community & $1.0(0.8-1.2)$ & .747 & & \\
\hline
\end{tabular}

were upstaged $79.4 \%$ of the time. Tumor size was also associated with upstaging: for tumors 3 to $4 \mathrm{~cm}$, the rate of upstaging was $49.2 \%$; for tumors 4 to $5 \mathrm{~cm}$, the rate of upstaging was $58.1 \%$; and for tumors $>5 \mathrm{~cm}$, the rate of upstaging was $55.5 \%$. Tumor size $>4 \mathrm{~cm}$ and presence of lymphovascular invasion placed the patients above the threshold for benefit of induction chemoradiation, even when perioperative mortality for that strategy was doubled within our model. Univariable and multivariable analyses confirmed these variables were significantly associated with upstaging (all $P$ values $<.0001$ ) versus same- or downstaging, and the results of these analyses and corresponding odds ratios can be seen in Table 4. A summary of the results of these analyses can be seen in Table 5 .

\section{DISCUSSION}

Our decision analysis allows us to integrate the best available clinical evidence for the role of induction therapy in patients with cT2N0 disease. Our baseline model demonstrated that, overall, patients with cT2N0 cancer who underwent upfront esophagectomy have an estimated survival benefit of approximately 2 to 3 months compared with patients who underwent induction therapy followed by esophagectomy. This benefit is likely due to the effect of the true lower-stage patients in our model. Survival for patients with disease staged less than pT2N0 is better with upfront surgery ${ }^{4}$ due to avoidance of unnecessary and likely harmful treatment in this population that could cause deconditioning, chemoradiation-related morbidity 
TABLE 5. Summary of conclusions and supporting results

\begin{tabular}{|c|c|c|}
\hline Analysis & Conclusion & Supporting results \\
\hline Baseline decision analysis model & $\begin{array}{l}\text { Survival is similar between upfront surgery } \\
\text { and induction chemoradiation, with a small } \\
\text { expected survival benefit of } 2.5 \text { mo for } \\
\text { upfront surgery }\end{array}$ & $\begin{array}{l}\text { Upfront esophagectomy yields an expected } \\
\text { overall survival of } 48.3 \text { mo compared with } \\
45.9 \text { mo for induction chemoradiation }\end{array}$ \\
\hline Sensitivity analysis of diagnostic uncertainty & $\begin{array}{l}\text { Induction chemoradiation is expected to yield } \\
\text { a survival advantage within the published } \\
\text { range of esophageal ultrasound } \\
\text { understaging for cT2N0 disease (ie, } 32 \% \text { - } \\
65 \% \text { ) }\end{array}$ & $\begin{array}{l}\text { The threshold for benefit is with a probability } \\
\text { of upstaging of }>48.1 \% \text { (see Figure } 2 \text { ) }\end{array}$ \\
\hline $\begin{array}{l}\text { Descriptive analysis of NCDB patient } \\
\text { characteristics by true stage }\end{array}$ & $\begin{array}{l}\text { Patients with a tumor grade } 3 \text { or } 4 \text {, tumor } \\
\text { size }>3 \mathrm{~cm} \text {, or presence of lymphovascular } \\
\text { invasion would be expected to gain a } \\
\text { survival benefit from induction } \\
\text { chemoradiation }\end{array}$ & $\begin{array}{l}\text { With any one of these features, the proportion } \\
\text { of patients who were upstaged was above } \\
\text { the threshold for benefit of } 48.1 \% \text { (see } \\
\text { Table 3) }\end{array}$ \\
\hline $\begin{array}{l}\text { Univariable and multivariable analysis of } \\
\text { NCDB patient characteristics }\end{array}$ & $\begin{array}{l}\text { Tumor grade, tumor size, and presence of } \\
\text { lymphovascular invasion were found to be } \\
\text { associated with risk of pathologic } \\
\text { upstaging }\end{array}$ & $\begin{array}{l}\text { These features were significant on } \\
\text { univariable and multivariable analysis (all } \\
P \text { values }<.0001 \text { ) (see Table } 4 \text { ) }\end{array}$ \\
\hline
\end{tabular}

NCDB, National Cancer Database.

or mortality, treatment delay, and potentially increased surgical complications and mortality. This result demonstrates that identification of higher-risk patients for induction therapy would be expected to yield better results than taking a uniform approach to this group.

Our findings also show that the theoretical threshold of EUS understaging (or pathologic upstaging) at which induction chemoradiation becomes beneficial is within the published range of diagnostic uncertainty. The results from this decision analysis can be used to guide clinical recommendations for a patient with cT2N0 cancer based on whether the patient's probability of pathologic upstaging is $>48.1 \%$. Our analysis of tumor factors from the NCDB identified several high-risk features that should raise suspicion for a true higher pathologic stage. It would be reasonable to routinely use induction chemoradiation for patients with any 1 of the following features placing a patient above the threshold for benefit: tumor grade 3 or 4 , presence of lymphovascular invasion, or tumor size $>3 \mathrm{~cm}$. Tumor size can be estimated from an endoscopic exam and grade can often be assessed from a routine biopsy. Identification of lymphovascular invasion may depend on the size and quality of a biopsy in addition to pathologic staining techniques, ${ }^{16,21,22}$ but can occasionally be determined preoperatively, ${ }^{23,24}$ and when available may be very helpful in guiding treatment.

Other universally available clinical features can also be considered in the application of our model. Specifically, dysphagia and positron emission tomography standardized uptake values. Although no studies have explicitly examined the probability of upstaging in cT2N0 patients with dysphagia, several series on resectable esophageal cancer have shown the presence of dysphagia to have a specificity for "at least T3 disease" of $>88 \%{ }^{25-27}$ Similarly, studies examining positron emission tomography standardized uptake values have not been done exclusively in patients with cT2N0 disease; however, a maximum standardized uptake value $>2.5$ in the primary tumor has been associated with presence of nodal disease. ${ }^{28,29}$ Presence of these factors will likely place a patient with cT2N0 disease above the $48.1 \%$ threshold for benefit of induction chemoradiation and should be considered during clinical decision making about the best treatment strategy.

This study has some limitations that should be considered. First, data for the model were derived from existing published studies and relied, at least partially, on retrospective reviews that may be influenced by selection bias. Patients who received adjuvant therapy likely had a smooth operative course and were healthy enough to tolerate this treatment. There may also be surgeon bias in selecting low-risk patients for upfront surgery and high-risk patients for induction therapy that may influence survival estimates for the pathologically staged subgroups. We used data from randomized controlled trials where available. However, these data may also be biased by the performance status needed to enroll in such trials: progression to inoperability after induction therapy as well as the average time interval from neoadjuvant treatment to surgery may be underestimated when considering that a patient with esophageal cancer may be frail. Second, for some survival data points we 
relied on cohorts that were quite similar to, but not exact matches for those in our model. One example is the use of survival data for patients undergoing planned definitive chemoradiation as the expected survival for patients unable to undergo an operation after induction therapy. However, the same factors that made these patients nonoperative candidates would likely result in shortened expected overall survival compared with those with fewer comorbidities, and therefore we believed this group was appropriately representative. Additionally, other articles have reported proportionally higher percentages of chemoradiationrelated mortality, sometimes up to $4 \%{ }^{30}$ These proportions occurred in studies with smaller sample sizes and the absolute number of deaths remained low. Therefore, we retained the lower estimated prevalence of death related to chemoradiation. Finally, all patients in the NCDB may not have received the CROSS regimen of induction therapy, which has low morbidity and mortality, and this may influence survival estimates. When considering these limitations, it is worth noting that in development and testing of our decision tree, none of these individual factors were primary drivers of the model outcome.

We believe these limitations are significantly outweighed by several major strengths of our approach. We incorporated the best-available existing evidence into a model that can inform clinical practice, with both the information provided from the baseline model as well as through our sensitivity analyses. We supplemented published data with primarily derived outcomes for specific pathologic subgroups using a large, validated dataset, obtaining survival information reflecting real-world, practice-based outcomes. Through sensitivity analyses, we were able to identify the drivers of our model and the threshold at which each treatment paradigm will confer improved survival. We analyzed clinical and pathologic features that would identify patients at high risk of upstaging, and consideration of these features in the context of our sensitivity analysis (Figure 2) allows for estimation of the expected survival benefit of induction chemoradiation for an individual. This may be helpful for providers in making treatment recommendations and risk-benefit discussions with patients. Additionally, our model implicitly accounts for patients who had a margin positive resection and postoperative complications-both of which would be expected to confer a worse overall survival with or without induction therapy. Rates for these outcomes were within similar ranges across publications used for our model input, and the estimated median survival incorporates patients with these poor prognostic factors. These features of our model allow for broad applicability.

\section{CONCLUSIONS}

This decision analysis provides evidence that induction chemoradiation improves survival in patients with cT2N0 esophageal cancer if the probability of EUS understaging is $>48.1 \%$. Upfront surgery is preferred when the error rate of EUS is below this threshold. Several clinical factors, including high tumor grade, presence of lymphovascular invasion, large tumor size, presence of dysphagia, or positron emission tomography standardized uptake value $>2.5$ increase the probability of EUS understaging and consequent pathologic upstaging and can guide a clinician to consider induction therapy. Although additional patient-specific factors must be considered with any clinical decision making, this study helps to provide guidance in the initial management of cT2N0 esophageal cancer in the face of the inherent diagnostic uncertainty of EUS staging (Table 5).

\section{Conflict of Interest Statement}

Authors have nothing to disclose with regard to clinical support.

\section{References}

1. National Cancer Institute, Surveillance, Epidemiology and ERP. Cancer of the Esophagus - Cancer Stat Facts. Cancer Statistics. https://seer.cancer.gov/ statfacts/html/esoph.html. Accessed June 19, 2017.

2. Rice TW, Blackstone EH, Rusch VW. 7th edition of the AJCC cancer staging manual: esophagus and esophagogastric junction. Ann Surg Oncol. 2010;17: 1721-4.

3. Mariette C, Dahan L, Mornex F, Maillard E, Thomas PA, Meunier B, et al. Surgery alone versus chemoradiotherapy followed by surgery for stage I and II esophageal cancer: final analysis of randomized controlled phase III trial FFCD 9901. J Clin Oncol. 2014;32:2416-22.

4. Rice TW, Mason DP, Murthy SC, Zuccaro G Jr, Adelstein DJ, Rybicki LA, et al. T2NOM0 esophageal cancer. J Thorac Cardiovasc Surg. 2007;133:317-24.

5. van Hagen P, Hulshof MCCM, van Lanschot JJB, Steyerberg EW, van Berge Henegouwen MI, Wijnhoven BPL, et al. Preoperative chemoradiotherapy for esophageal or junctional cancer. $N$ Engl J Med. 2012;366:2074-84.

6. Chen W-H, Chao Y-K, Chang H-K, Tseng CK, Wu YC, Liu YH, et al. Long-term outcomes following neoadjuvant chemoradiotherapy in patients with clinical T2N0 esophageal squamous cell carcinoma. Dis Esophagus. 2012;25:250-5.

7. Dolan JP, Kaur T, Diggs BS, Luna RA, Sheppard BC, Schipper PH, et al. Significant understaging is seen in clinically staged T2N0 esophageal cancer patients undergoing esophagectomy. Dis Esophagus. 2016;29:320-5.

8. Speicher PJ, Ganapathi AM, Englum BR, Onaitis MW, D'Amico TA, Berry MF. Induction therapy does not improve survival for clinical stage T2N0 esophageal cancer. J Thorac Oncol. 2014;9:1195-201.

9. Zhang JQ, Hooker CM, Brock MV, Shin J, Lee S, How R, et al. Neoadjuvant chemoradiation therapy is beneficial for clinical stage T2 N0 esophageal cancer patients due to inaccurate preoperative staging. Ann Thorac Surg. 2012;93:429-35.

10. Crabtree TD, Yacoub WN, Puri V, Azar R, Zoole JB, Patterson GA, et al. Endoscopic ultrasound for early stage esophageal adenocarcinoma: implications for staging and survival. Ann Thorac Surg. 2011;91:1509-15.

11. Stiles BM, Mirza F, Coppolino A, Port JL, Lee PC, Paul S, et al. Clinical T2T3N0M0 esophageal cancer: the risk of node positive disease. Ann Thorac Surg. 2011;92:491-6.

12. Crabtree TD, Kosinski AS, Puri V, Burfeind W, Bharat A, Patterson GA, et al Evaluation of the reliability of clinical staging of T2 N0 esophageal cancer: a review of the Society of Thoracic Surgeons database. Ann Thorac Surg. 2013;96: 382-90.

13. Hofstetter W. Treatment of clinical T2N0M0 esophageal cancer. Ann Surg Oncol. 2014;21:3713-4.

14. Hardacker TJ, Ceppa D, Okereke I, Rieger KM, Jalal S, LeBlanc JK, et al. Treatment of clinical T2N0M0 esophageal cancer. Ann Surg Oncol. 2014;21:3739-43.

15. Tekola BD, Sauer BG, Wang AY, White GE, Shami VM. Accuracy of endoscopic ultrasound in the diagnosis of T2N0 esophageal cancer. J Gastrointest Cancer. 2014;45:342-6.

16. Samson P, Puri V, Robinson C, Carpenter D, Broderick S, Kreisel D, et al. Clinical T2N0 esophageal cancer: identifying pretreatment characteristics associated 
with pathologic upstaging and the potential role for induction therapy. Ann Thorac Surg. 2016;101:2102-11.

17. Abrams JA, Buono DL, Strauss J, McBride RB, Hershman DL, Neugut AI. Esophagectomy compared with chemoradiation for early stage esophageal cancer in the elderly. Cancer. 2009;115:4924-33.

18. Lapar DJ, Stukenborg GJ, Lau CL, Jones DR, Kozower BD. Differences in reported esophageal cancer resection outcomes between national clinical and administrative databases. J Thorac Cardiovasc Surg. 2012;144: 1152-7.

19. Medical Research Council Oesophageal Cancer Working Group. Surgical resection with or without preoperative chemotherapy in oesophageal cancer: a randomised controlled trial. Lancet. 2002;359: 1727-33.

20. Fiorica F, Di Bona D, Schepis F, Licata A, Shahied L, Venturi A, et al. Preoperative chemoradiotherapy for oesophageal cancer: a systematic review and metaanalysis. Gut. 2004;53:925-30.

21. Saad RS, Lindner JL, Liu Y, Silverman JF. Lymphatic vessel density as prognostic marker in esophageal adenocarcinoma. Am J Clin Pathol. 2009;131: 92-8.

22. Goscinski MA, Nesland JM, Giercksky K-E, Dhakal HP. Primary tumor vascularity in esophagus cancer. CD34 and HIF1- $\alpha$ expression correlate with tumor progression. Histol Histopathol. 2013;28:1361-8.

23. Cen P, Hofstetter WL, Lee JH, Ross WA, Wu TT, Swisher SG, et al. Value of endoscopic ultrasound staging in conjunction with the evaluation of lymphovascular invasion in identifying low-risk esophageal carcinoma. Cancer. 2008;112: 503-10.
24. Cen P, Hofstetter WL, Correa AM, Wu TT, Lee JH, Ross WA, et al. Lymphovascular invasion as a tool to further subclassify T1b esophageal adenocarcinoma. Cancer. 2008;112:1020-7.

25. Fang TC, Oh YS, Szabo A, Khan A, Dua KS. Utility of dysphagia grade in predicting endoscopic ultrasound T-stage of non-metastatic esophageal cancer. Dis Esophagus. 2016;29:642-8.

26. Ripley RT, Sarkaria IS, Grosser R, Sima CS, Bains MS, Jones DR, et al. Pretreatment dysphagia in esophageal cancer patients may eliminate the need for staging by endoscopic ultrasonography. Ann Thorac Surg. 2016;101:226-30.

27. Schlansky B, Dimarino AJ, Loren D, Infantolino A, Kowalski T, Cohen S. A survey of oesophageal cancer: pathology, stage and clinical presentation. Aliment Pharmacol Ther. 2006;23:587-93.

28. I HS, Kim S-J, Kim IJ, Kim K. Predictive value of metabolic tumor volume measured by 18 F-FDG PET for regional lymph node status in patients with esophageal cancer. Clin Nucl Med. 2012;37:442-6.

29. Shin S, Kim HK, Choi YS, Kim K, Shim YM. Clinical stage T1-T2NOM0 oesophageal cancer: accuracy of clinical staging and predictive factors for lymph node metastasis. Eur J Cardio-Thoracic Surg. 2014;46:274-9.

30. Tepper J, Krasna MJ, Niedzwiecki D, Hollis D, Reed CE, Goldberg R, et al. Phase III trial of trimodality therapy with cisplatin, fluorouracil, radiotherapy, and surgery compared with surgery alone for esophageal cancer: CALGB 9781. J Clin Oncol. 2008;26:1086-92.

Key Words: esophageal cancer, endoscopic ultrasound, induction chemoradiation, esophagectomy 
Survival of Patients Undergoing Induction Therapy Prior to Esophagectomy

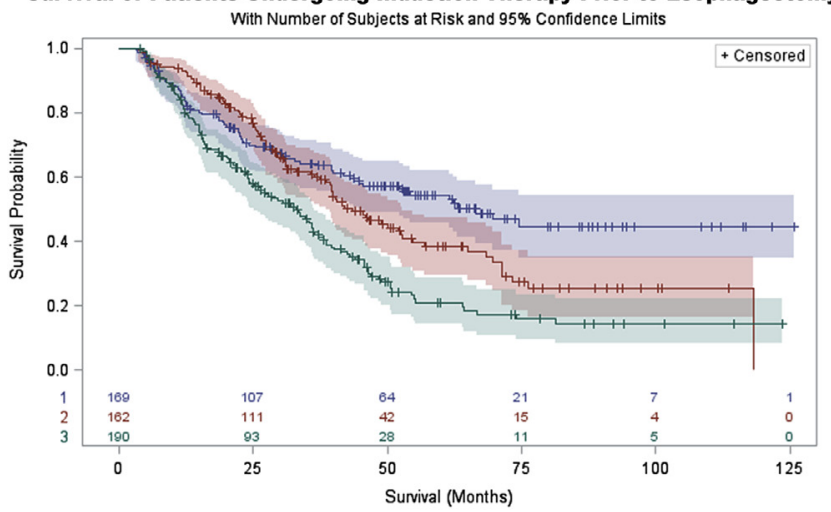

A

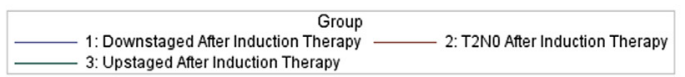

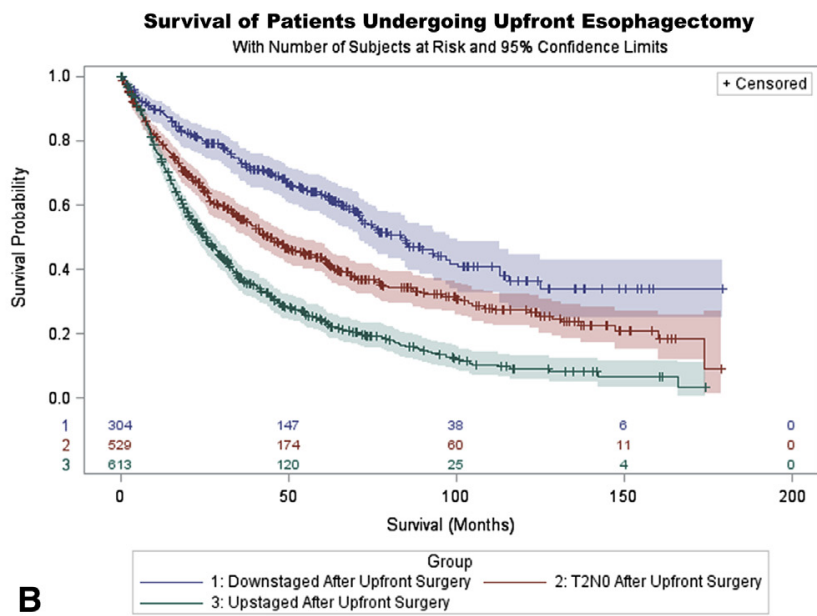

B
1: Downstaged After Upfront Surgery

FIGURE E1. Kaplan-Meier survival curves determined through use of Kaplan-Meier analysis for input into our decision tree. A, Median survival for patients receiving induction chemoradiation. B, Median survival for patients undergoing upfront esophagectomy after clinical T2N0 diagnosis. Relevant median survivals that were included in our model are detailed in Table 1. 